$A R T I C U L O P B$

IN VES TI I G AC I Ó

C I E N T I F I I C A Y Y

$T E C N O L O O B G I C A$

\section{UNIVERSIDAD E IDENTIDAD. USOS Y APROPIACIONES DE LOS SIGNOS VISUALES Y REPRESENTACIONES SOCIALES DE LA Universidad de Cartagena}

\author{
UNIVERSITY AND IDENTITY. USES AND APPROPRIATIONS \\ OF VISUAL SIGNS AND SOCIAL REPRESENTATIONS OF THE \\ UNIVERSITY OF CARTAGENA
}

Por: Jorge Enrique Matson Carballo

Indira de la Concepción Romero Peñaranda

*JORGE ENRIQUE MAT-

SON CARBALLO

Cartagena de Indias

Colombia.

Comunicador Social-

periodista de la Uni-

versidad Autónoma del

Caribe, Colombia. Más-

ter en Comunicación de

la Universidad Inter-

nacional de Andalucía,

España. Especialista en

Estudios Políticos del

convenio Universidad

Sergio Arboledarsidad

Sergio Arboleda-Escuela

Naval Almirante Padilla,

Colombia. Magíster en

Educación, SUE Caribe-

Universidad de Cartage-

na, Colombia. Asesor en

Calidad y Comunicación

de la Universidad de Car-

tagena y docente de Cá-

tedra en el Programa de

Comunicación Social de

la Universidad de Carta-

gena. Correo electrónico:

Jemca2008@yahoo.es

**INDIRA DE LA

CONCEPCIÓN ROMERO PENNARANDA

Cartagena de Indias,

Colombia

Diseñadora Gráfica de la Universidad de Bogo-

tá Jorge Tadeo lozano,

Colombia. Especialista

en Educación Artística

de la Universidad del Atlántico. Magíster en Educación SuE CaribeUniversidad de Cartagena, Colombia Jefe de gena, Colombia. Jefe del Departamento de Cienclas Humanas, Artes y Diseño, de la Universidad Jorge Tadeo Lozano, Seccional del Caribe. Correo electrónico: indianadelac@gmail.com

\begin{abstract}
RESUMEN
Las instituciones de Educación Superior, IES, se enfrentan a grandes retos generados en la configuración de la denominada Sociedad del Conocimiento, por los cuales ven afectadas las dinámicas de cambio de sus propuestas educativas. Ante tal panorama, la Universidad de Cartagena, debe instrumentar mecanismos para sobrevivir ante la visión tecnocrática y economicista persistente. Uno de estos posibles mecanismos por los que se aboga, es la revisión de la identidad de la IES, con la intención de fortalecer la cultura institucional y a partir de allí, las identidades nacionales. Al existir diversas miradas sobre el fenómeno de la identidad, se considera revisar, la de la Universidad de Cartagena, desde la óptica de los signos visuales y representaciones sociales. Estos se manifiestan configurando la identidad universitaria y facilitan identificar puntos de encuentro entre las subjetividades presentes en sus estamentos constitutivos internos, y se enriquecen a partir de la memoria y las prácticas socioculturales cotidianas.
\end{abstract}

Palabras clave: Identidad, signos visuales, representaciones sociales, Universidad de Cartagena, usos y apropiaciones.

\begin{abstract}
Institutions of higher education, IES, face great challenges generated in the configuration of the so-called knowledge society. These tend to be character curriculum, technological, political, economic and cultural, in such a way that, the IES affected the dynamics of change of his educational proposals; circumstance, which impacts on the social function and the nature of the institution. Against such background, the University of Cartagena, must implement mechanisms to survive in persistent technocratic and economistic vision. One of the mechanisms by which is advocated is the revision of the identity of the IES, in its instances of self-assessment, with the intention of strengthening institutional and from there, culture national identities. There are different perspectives on the phenomenon of identity in a world communicating by signs, is considered to check the identity of the University of Cartagena from the standpoint of Visual signs and social representations. These manifest themselves by configuring the University identity, make it easy to identify meeting points between present in its internal constituent strata subjectivities and enrich themselves from memory and everyday socio-cultural practices.
\end{abstract}

Keywords: Identity, visual signs, social representations, University of Cartagena, uses and appropriations. 


\section{I.Introducción}

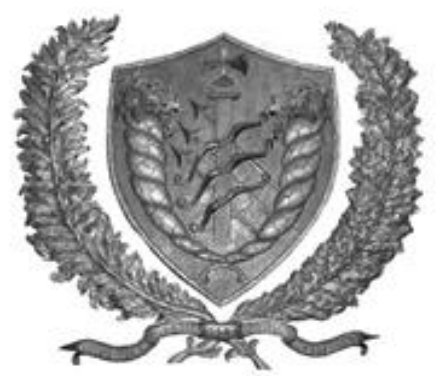

Fotografía: Mario Lorduy Benedetti/2008

"[...] La realidad auténtica la poseemos únicamente en los hechos de la conciencia que se nos dan en la experiencia interna"

(Dilthey, 1978, p. 16).

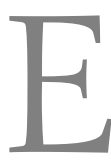

l presente artículo, expone los resultados de La investigación

"Universidad e identidad. Usos y apropiaciones de los signos visuales y representaciones sociales de la Universidad de Cartagena", la cual, se planteó con el objetivo de conocer cómo estos usos y las apropiaciones dan cuenta de la configuración actual de identidad de la Universidad de Cartagena. El abordaje del tema tuvo un carácter indagatorio con visión prospectiva para la universidad latinoamericana, se constituyó la idea de que para que las Instituciones de Educación Superior, en especial, la Universidad de Cartagena puedan sobrevivir al fenómeno de la empresarialización, deben tener conciencia de su identidad en relación con el contexto, que según la Declaración de la Conferencia Regional de la Educación Superior en América Latina y el Caribe-[CRES] en el 2008, se gestiona bajo los parámetros de la Organización Mundial del Comercio. Ver tabla 1.

Tabla 1. Retos de la universidad latinoamericana

\begin{tabular}{|c|c|}
\hline Ámbito Internacional & Ámbito Nacional/Local \\
\hline $\begin{array}{c}\text { Sociedad del conocimiento/ } \\
\text { Economía del conocimiento }\end{array}$ & $\begin{array}{c}\text { Ampliación de cobertura y } \\
\text { retención estudiantil }\end{array}$ \\
\hline $\begin{array}{c}\text { Internacionalización de la Educación } \\
\text { Superior (Educación transfronteriza) }\end{array}$ & $\begin{array}{c}\text { Mejoramiento de la calidad } \\
\text { en la Educación Superior }\end{array}$ \\
\hline Redes de investigación & Eficacia del sector educativo \\
\hline $\begin{array}{c}\text { Acreditación internacional de la } \\
\text { Institución de Educación Superior }\end{array}$ & $\begin{array}{c}\text { Transparencia y rendición de cuentas en } \\
\text { el sector educativo }\end{array}$ \\
\hline $\begin{array}{c}\text { Propiedad intelectual } \\
\text { (Patentes y derechos de autor) }\end{array}$ & \begin{tabular}{c} 
Desarrollo de ciencia y tecnología \\
\hline Educación virtual
\end{tabular} \\
\hline \multirow{2}{*}{$\begin{array}{c}\text { Espacios Latinoamericanos y del Caribe } \\
\text { Europeo para la Educación Superior }\end{array}$} & $\begin{array}{c}\text { Tratado de Libre Comercio y tecnificación } \\
\text { de la propuestas curriculares }\end{array}$ \\
\cline { 2 - 2 } & Reforma de la Ley 30 de 1992 \\
\hline
\end{tabular}

Fuente: Investigación "Universidad e identidad. Usos y apropiaciones de los signos visuales y representaciones sociales de la Universidad de Cartagena". Autores, Indira Romero Peñaranda y Jorge Matson Carballo, 2013. 


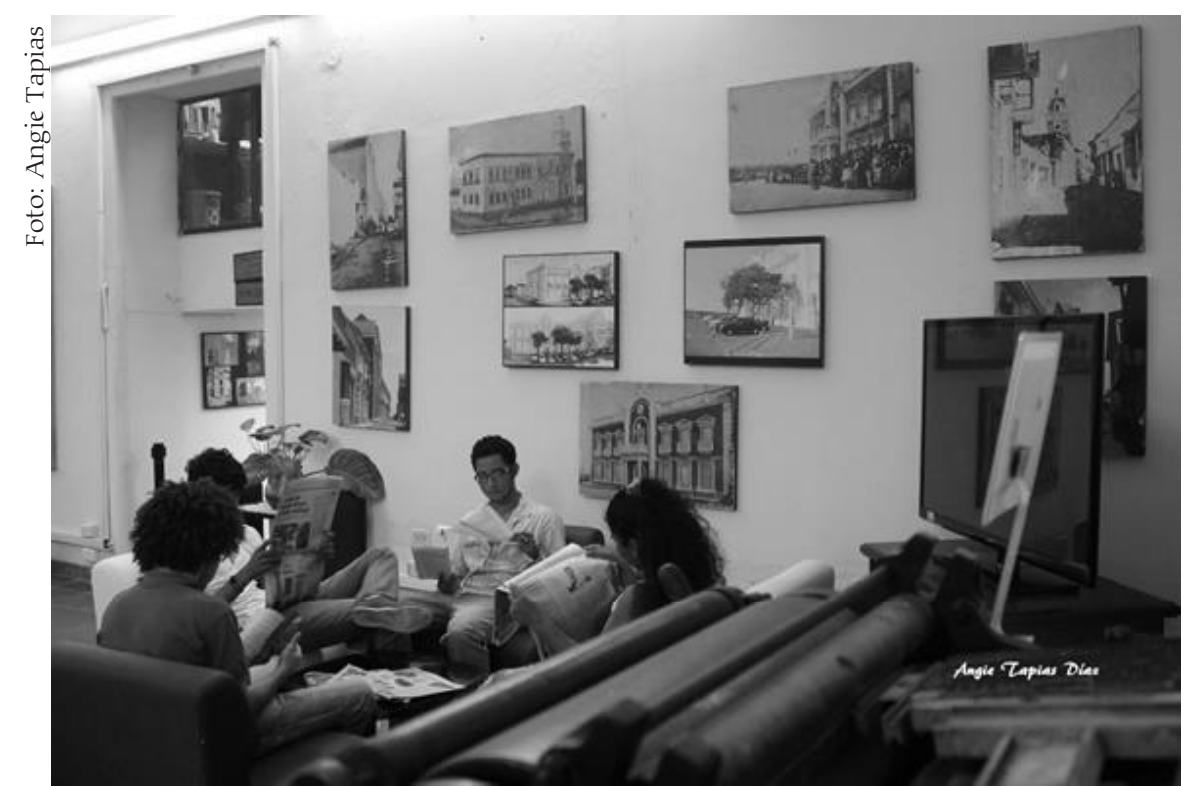

El enfoque de esta investigación, aportó al contexto establecido en la investigación iniciada en 1996 por Jorge Matson Carballo en "El escudo de la Universidad de Cartagena: símbolo de identidad", publicado en 2004, en el volumen 2 de la colección "La Cátedra de la Universidad de Cartagena, mirar su historia para construir su futuro". La preocupación inicial de Jorge Matson sobre una identidad institucional no apropiada, lo llevó a identificar la vinculación de la Universidad con los orígenes de la nacionalidad colombiana en el Congreso de Cúcuta de 1821. El abordaje propuesto desde el actual proyecto, aportó una lectura del fenómeno desde un nivel propositivo más abarcador, el de "signo"; considerando así, a los signos visuales y las representaciones sociales como los elementos fundamentales del problema en relación con la identidad universitaria para el análisis de los fenómenos de la cultura institucional en la IES, aportando una mirada diferente a la identificada en el estado del arte, en relación con el hallazgo de trabajos en Iberoamérica, Estados Unidos y, Reino Unido, entre otros, que plantean el problema de la identidad desde estudios tradicionales para las organizaciones.

Para comprender mejor la situación se orientó la investigación bajo el supuesto que: a) La identidad es una configuración, entendida esta última como una coyuntura en el espacio-tiempo. Para ello, se consultó al sociólogo jamaiquino Stuart Hall, "las identidades [...] se constituyen dentro de la representación y no fuera de ella (Hall, 2003, P17); al antropólogo francés Marc Augé, y los documentos técnicos del Programa de las Naciones Unidas para el Desarrollo PNUD. b) La configuración está dada por representaciones sociales, y para ello, se consultó a Enrique Toledo De la Garza, sociólogo mejicano de la escuela de García Canclini, quien plantea que la configuración responde a una nueva articulación epistemológica que intenta fundamentar el conocimiento de una realidad en permanente transformación, vista como "una coyuntura del tiempo presente en el espacio de posibilidades", una realidad vista en un nivel 
profundo de comprensión de la cotidianidad, con el ánimo de evidenciarlo como oportunidad de cambio (De La Garza, 2001). c) Las representaciones sociales, se originan y sostienen por los usos y apropiaciones de los signos visuales de la comunidad académica. Se utilizó como referente la teoría del psicólogo social francés Sergei Moscovici y de la filósofa, de la misma escuela, Denise Jodelet, quien hace referencia a las representaciones sociales, como un conocimiento profundo que surge de la observación cotidiana y que se constituye a partir de las experiencias personales, pero también de las informaciones y conocimientos (Jodelet. 1976, P.473). En palabras de Moscovici (2002), estas, "permiten conocer cómo los grupos sociales conceptualizan un objeto material o simbólico". Esto es, la manera cómo se construye y otorgan atributos representativos a esas situaciones cotidianas. d) Los signos visuales, son una categoría abarcadora que permite incluir, el ícono, el índice y el símbolo, para lo cual se tomó en consideración la teoría del signo del filósofo norteamericano Charles S. Peirce. El estudio de los signos, necesariamente hace referencia a estudiar la interacción social. En este horizonte, la flexibilidad que brinda la semiótica con la teoría de Charles S. Peirce, ayuda a conocer el signo desde una perspectiva integral, en el cual la participación, la interpretación y la acción se mueven en un sentido holístico al poder relacionar distintos ámbitos del conocimiento en una propuesta no lineal de pensamiento. Y por último, e) las aplicaciones y percepciones de los signos visuales, manifiestan la dimensión socio-cultural de la Universidad. Para sostener este supuesto, se consultaron los documentos técnico-conceptuales de la UNESCO en materia de educación superior y desarrollo.

\section{Métodología}

7 ara el diseño de la investigación, partimos de los usos y apropiaciones de los signos visuales y representaciones de la Universidad de Cartagena que hacen los miembros de esta institución y que al mismo tiempo dan cuenta de su configuración de identidad. Lo anterior en atención a los supuestos ontológico (identidad universitaria-representaciones sociales), epistemológico (configuración en contexto), metodológico (interpretación de usos y apropiaciones de signos visuales y representaciones sociales) y a las fuentes primarias y secundarias de información. Se determinó el método cualitativo siguiendo los planteamientos de Corbi y Strauss (Corbi y Strauss, 2002, pp. 13-14) quienes señalan que "la investigación cualitativa tiene componentes variados, es decir, las fuentes a usar y los procedimientos para interpretar, pueden ser diferentes (siendo) uno de estos, la conceptualización". Los autores explican que, "al hecho de conceptualizar, reducir, elaborar y 
Fuente: Investigación: Universidad e identidad. Usos y apropiaciones de los signos visuales y representaciones sociales de la Universidad de Cartagena. Autores Indira Romero Peñaranda y Jorge Matson Carballo. 2013. relacionar datos se le suele denominar codificar. [...] Se refieren a una teoría derivada de datos recopilados de manera sistemática y analizada por medio de un proceso de investigación. [...] Más bien, comienza con un área de estudio y permite que la teoría sea derivada de los datos" (Corbi y Strauss, 2002, pp. 13-14). Por ello, para el desarrollo se diseñó el método de la siguiente forma. Ver tabla 2.

\abla 2. Método de investigación

\begin{tabular}{|r|l|}
\hline Enfoque & Hermenéutico - fenomenológico \\
\hline Tipo & Descriptivo - exploratorio \\
\hline Perspectiva & Holística \\
\hline Estrategia & Análisis de contenido \\
\hline \multirow{2}{*}{$\begin{array}{r}\text { Técnicas de recolección } \\
\text { de la información }\end{array}$} & $\begin{array}{l}\text { Grupo focal ( seis grupos, con participantes entre 12 } \\
\text { servidores públicos no docentes. }\end{array}$ \\
\cline { 2 - 3 } & Análisis de documentos \\
\cline { 2 - 3 } & Observación participante \\
\hline Instrumentos & $\begin{array}{l}\text { Guion para grupo focal, matrices para sistem- } \\
\text { atización, Software Atlas Ti }\end{array}$ \\
\hline
\end{tabular}

La aplicación de los instrumentos indistintamente de sus características se hizo considerando cuatro etapas: la planeación, la recolección de la información, el análisis de la información y por último la reflexión.

Ante la complejidad del estudio, se optó por trabajar con los grupos representativos que de mejor forma dieran cuenta de las transformaciones en la cultura institucional, como participantes del diseño de un contexto en constante evolución y que afecta las dinámicas internas de relaciones, de los que en el momento de aplicación de los instrumentos hacen parte activa de la configuración de la identidad Udeceista desde dentro. Así las cosas, se decidió trabajar con el estamento docente, el estamento estudiantil y el estamento de empleados públicos no docentes, seleccionados, bajo los siguientes criterios: a) permanencia en la institución; b) tiempo de servicio; c) ubicación espacial; d) conformación interdisciplinar: que los docentes y los estudiantes participantes representaran a diversas disciplinas del conocimiento; e) modalidad de estudio: diversas modalidades de acceso a la educación, específicamente la educación a distancia y postgrado; f) diversidad en grados de formación: que los estudiantes participantes cursasen preferiblemente de 
segundo a último período académico, y g) participación indistinta de diversos niveles jerárquicos sin considerar las funciones del personal administrativo. Surtida esta etapa, se escogieron ocho representantes por cada estamento. En la mayoría de los grupos, aumentó la participación, que osciló entre 10 y 12 participantes.

La investigación se llevó a cabo en la Universidad de Cartagena, en la ciudad de Cartagena de Indias, capital del Departamento de Bolívar en Colombia. Esta Universidad, fue fundada en 1827 su campus principal ocupa el edificio Claustro de San Agustín, que data del siglo XV, ubicado en el Centro amurallado de la Ciudad, declarada por la UNESCO en 1985, como Patrimonio Histórico de la Humanidad. Otro del los campus de la Universidad, ubicado en el Centro Histórico, es el Claustro de la Merced. Los demás campus, están ubicados en los barrios extramuros de la ciudad, Zaragocilla y Piedra de Bolívar. Es de resaltar que fue una de los primeros centros de estudio fundados en la nueva República y el primero de la costa Caribe colombiana, lo cual a lo largo de 187 años la ha convertido en un referente cultural en el proceso de conformación de la identidad regional.

La ubicación geográfica y la diversidad de su oferta formativa han sido elementos sustanciales a tener presente para la aplicación de las técnicas de investigación, especialmente para los grupos focales, ya que durante la aplicación de la prueba piloto de los mismos, emergió la exigencia por parte de los participantes que las sesiones fuesen desarrolladas en diversos campus, para romper con el centralismo de la institución. En consecuencia, se desarrollaron los diferentes grupos focales tanto en el Claustro de San Agustín, como en el Campus de la Salud en el barrio de Zaragocilla.

\section{Resultados}

- a primera parte, da cuenta de la configuración histórica de una idea de Universidad generada en la tradición discursiva, de uso y apropiación del escudo actual. La segunda parte, hace referencia a los hallazgos sobre los usos de los signos visuales y presenta las aplicaciones prácticas de los signos visuales en diferentes piezas de comunicación visual y la tercera parte, hace referencia, tanto a las apropiaciones de los signos visuales, como a las representaciones sociales, asociadas a la identidad universitaria. Ver tabla 3 
Fuente: Diseño de los autores basados en el documento Ideas educativas de Simón Bolívar de Rojas Armando (1998, p.98) citado por Vicente Lecuna, papeles de Bolívar (p. 309) y del documento Historia de la Universidad de Cartagena de Mario León Echeverría (1971, p. 59).
Tabla 3. Identidad histórica de la Universidad de Cartagena

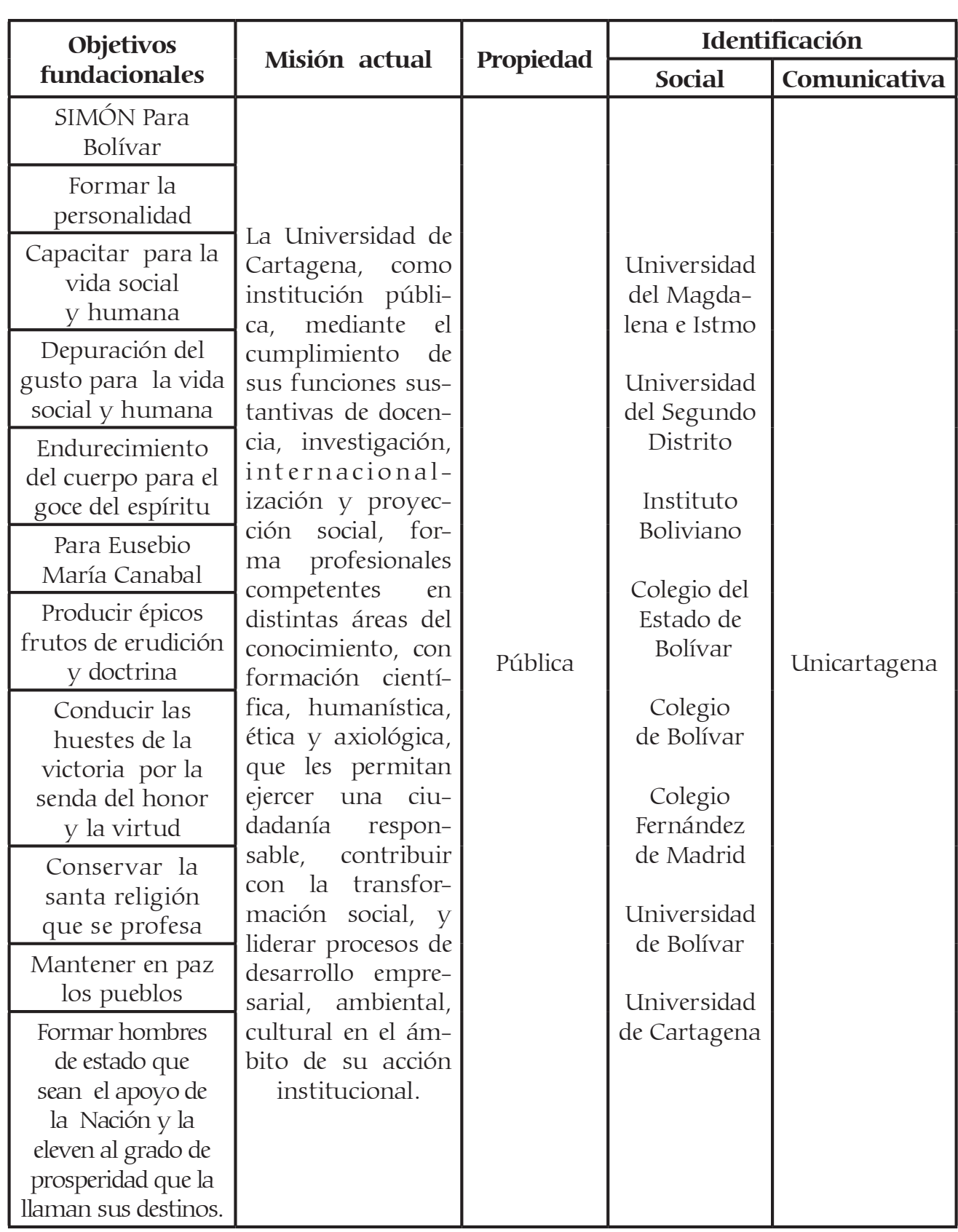

La tabla anterior, evidencia el discurso histórico que relaciona a la Universidad con los orígenes de la República, esta tradición adquiere mayor valor a partir de la oficialización del mismo, cuando en 1954 el rector Eduardo Lemaitre, acoge el escudo de la Gran Colombia como el de la Universidad de Cartagena. Se establece así una coherencia entre los ideales libertarios de los padres de la patria y los héroes de la independencia y la misión actual de la Universidad. 
Para comprender el escudo en su versión actual considerando su naturaleza de signo visual, el análisis se abordó desde las teorías de análisis del diseño planteadas por el investigador Wucius Wong, quien señala que "a diferencia del lenguaje hablado o escrito, cuyas leyes gramaticales están más o menos establecidas, el lenguaje visual carece de leyes obvias. Cada teórico del diseño puede poseer un conjunto de descubrimientos distintos por completo." (2001, p. 41). Bajo ese criterio, se obtuvo la siguiente caracterización. Ver tabla 4.

Tabla 4. Elementos de diseño: versión actual del escudo

\begin{tabular}{|c|c|c|}
\hline \multirow{4}{*}{$\begin{array}{c}\text { Elementos } \\
\text { conceptuales }\end{array}$} & Punto & $\begin{array}{l}\text { Se hace manifiesto, se puede apreciar en los elemen- } \\
\text { tos mínimos de forma circular, que se intercalan en- } \\
\text { tre las hojas que conforman la corona de laureles. }\end{array}$ \\
\hline & Línea & $\begin{array}{l}\text { El campo del escudo tiene demarcado su con- } \\
\text { torno con una línea de grosor uniforme, o borde } \\
\text { de forma orgánica y sinuosa, o lo que se conoce } \\
\text { en heráldica como trechor en el contorno. Estos, } \\
\text { tanto el campo como el trechor, conforman el } \\
\text { plano sobre el cual se disponen los cuernos de la } \\
\text { abundancia, las flechas, los arcos y las fasces. }\end{array}$ \\
\hline & Plano & $\begin{array}{l}\text { El mismo campo del escudo conforma un plano de } \\
\text { forma orgánica e irregular, que se dispone con la } \\
\text { mayor proporción de tamaño en toda la composición. }\end{array}$ \\
\hline & Volumen & $\begin{array}{l}\text { "En un diseño bi-dimensional, el volumen es iluso- } \\
\text { rio" (Wong, 2001, p. 42), en este sentido, las for- } \\
\text { mas orgánicas de los cuernos de la abundancia, las } \\
\text { flechas, los arcos y la fasce aparecen simulando vol- } \\
\text { umen, con los brillos propios del material metálico. }\end{array}$ \\
\hline \multirow{3}{*}{$\begin{array}{c}\text { Elementos } \\
\text { visuales }\end{array}$} & Forma & $\begin{array}{l}\text { Las formas presentes, tanto en las líneas, como } \\
\text { en planos y volúmenes, se caracterizan por ser } \\
\text { orgánicas, tales como las hojas que componen la } \\
\text { corona, los cuernos de la abundancia, las flechas y } \\
\text { la fasce. En el campo del escudo, es posible distin- } \\
\text { guir una figura con forma irregular, compuesta } \\
\text { por un contorno conformado por líneas rectas y } \\
\text { líneas sinuosas. }\end{array}$ \\
\hline & Medida & $\begin{array}{l}\text { Las medidas de cada uno de los componentes del } \\
\text { escudo son variadas. Se destacan por una mayor } \\
\text { magnitud el campo del escudo y la corona de hojas; } \\
\text { en una medida intermedia se encuentran los cuer- } \\
\text { nos de la abundancia, las flechas, los arcos y la fasce. } \\
\text { En una menor proporción, las perlas y la divisa. }\end{array}$ \\
\hline & Color & $\begin{array}{l}\text { Se destaca el color negro del campo que le imprime } \\
\text { profundidad a toda la composición, así mismo el } \\
\text { color verde de la corona de laureles, cobra gran im- } \\
\text { portancia al rodear este campo. Estos colores con- } \\
\text { trastan con el esmalte dorado de los objetos que se } \\
\text { disponen superpuestos flotando en el campo. }\end{array}$ \\
\hline
\end{tabular}




\begin{tabular}{|c|c|c|}
\hline & Textura & $\begin{array}{l}\text { Las superficies texturadas, se encuentran en las } \\
\text { nervaduras de las hojas de la corona de laureles. } \\
\text { Así mismo, una textura orgánica que permite } \\
\text { percibir un follaje en el remate de frutas y plantas } \\
\text { de los cuernos de la abundancia. También se en- } \\
\text { cuentra en la textura metálica que aparentan los } \\
\text { objetos que están sobre el campo del escudo. }\end{array}$ \\
\hline \multirow{4}{*}{$\begin{array}{l}\text { Elementos } \\
\text { de relación }\end{array}$} & Dirección & $\begin{array}{l}\text { La dirección con la que se disponen los elemen- } \\
\text { tos en el escudo es central. La ubicación al tener } \\
\text { este carácter central permite percibir un escudo } \\
\text { estable, regular y equilibrado. }\end{array}$ \\
\hline & Posición & $\begin{array}{l}\text { La posición de los elementos que conforman el } \\
\text { escudo es simétrica. La simetría hace referencia a } \\
\text { cuando una "Figura o forma con su imagen refle- } \\
\text { ja en disposición bilateral"(Wong, 2001, p. 348). } \\
\text { Esta característica está íntimamente ligada a la de } \\
\text { dirección y fortalece los conceptos de jerarquía y } \\
\text { poder, así como la idea de la perfección. }\end{array}$ \\
\hline & Espacio & $\begin{array}{l}\text { El escudo de la Universidad de Cartagena está con- } \\
\text { formado por dos grupos de elementos fusionados: } \\
\text { El primer grupo lo conforman el campo con los } \\
\text { objetos metálicos superpuestos en él y el segundo } \\
\text { incluye la corona de laureles y la divisa. }\end{array}$ \\
\hline & Gravedad & $\begin{array}{l}\text { El distanciamiento entre los dos bloques de elemen- } \\
\text { tos, permite tener una sensación de suficiencia tal } \\
\text { del espacio flotante que se reafirma la percepción de } \\
\text { equilibrio que ya se ha logrado con los otros ele- } \\
\text { mentos. No es posible sentir inestabilidad en la com- } \\
\text { posición, pues los pesos visuales se compensan. }\end{array}$ \\
\hline $\begin{array}{l}\text { Elementos } \\
\text { prácticos }\end{array}$ & Representación & $\begin{array}{l}\text { Laureles: ramas de arbusto de laurel, hojas perennes } \\
\text { rodean el campo que contiene la figura simbólica. } \\
\text { Campo: "cuerpo/espacio de forma suiza de color } \\
\text { negro que da profundidad y sensación de volu- } \\
\text { men a las figuras simbólicas que contiene". } \\
\text { Divisa: en el escudo, recoge el nombre de marca "Uni- } \\
\text { versidad de Cartagena", y } 1827 \text { como año de fundación. } \\
\text { Cornucopias: cuerno curvo y hueco de a par vol- } \\
\text { teados hacia arriba que contienen según la Ley del } \\
4 \text { de octubre de } 1821 \text { de acuerdo con el artículo } \\
11 \text { de la Ley Fundamental del Congreso de Cúcuta, } \\
\text { "frutos y flores de los países fríos, templados y } \\
\text { cálidos. Figura de origen mítico griego asociado a } \\
\text { Zeus y Hércules, apropiado por los romanos. } \\
\text { Frutos y flores: en heráldica, figuras naturales, no son } \\
\text { perceptibles a simple vista, sin embargo, la bandera de } \\
\text { la Gran Colombia expuesta durante el Bicentenario } \\
\text { en la municipalidad de Caracas, deja ver claramente } \\
\text { una piña y una granada entre otras figuras naturales } \\
\text { poco deducibles. (Bolívar, 1983, p. } 737 \text { ). }\end{array}$ \\
\hline
\end{tabular}




\begin{tabular}{|c|c|}
\hline Representación & $\begin{array}{l}\text { Fasces colombianas: conjunto de } 12 \text { flechas uni- } \\
\text { das en un cilindro que son, sostenidas por la pre- } \\
\text { sión de una segur o hacha atravesada. Denomi- } \\
\text { nada así según la Ley del } 4 \text { de octubre de } 1821 \\
\text { del Congreso de Cúcuta. Es una representación } \\
\text { trasferida y adaptada del emblema de la primera } \\
\text { república francesa de } 1792 \text { (Matson, en "El escudo } \\
\text { de la Universidad de Cartagena, símbolo de iden- } \\
\text { tidad, pág. 169, 2004) cuyo origen se remonta } \\
\text { a las fasces romanas que eran enseñas de poder } \\
\text { portadas por los lictores, como aplicadores de la } \\
\text { justicia del consulado romano. } \\
\text { Arcos y flechas: tres arcos y tres flechas cruzados } \\
\text { en diagonal hacia arriba y separados; a diferencia, } \\
\text { del escudo aprobado por la Ley del } 4 \text { de octubre } \\
\text { de } 1821 \text {, que ordenó un emblema con "arcos y } \\
\text { flechas cruzados, atados con cinta tricolor en la } \\
\text { parte inferior. } \\
\text { Esmalte: se refiere a una sustancia material colo- } \\
\text { cada sobre la superficie de las figuras para repre- } \\
\text { sentar el acabado metálico en oro y los brillos de } \\
\text { las piezas del escudo. } \\
\text { Colores: verde: en heráldica sinople, sobre los lau- } \\
\text { reles y la divisa con brillos de esmalte en oro. } \\
\text { Negro: nn heráldica sable, en el campo }\end{array}$ \\
\hline Significado $^{1}$ & $\begin{array}{l}\text { Timbre, (laureles): gloria. Ornamento o adornos } \\
\text { exteriores del escudo. } \\
\text { Campo: "espacio comprendido dentro de las } \\
\text { líneas que limitan el mismo, es decir, a lo que } \\
\text { hemos definido antes como escudo, propiamente } \\
\text { dicho; y también se denomina campo al fondo } \\
\text { (...) Divisa: frase o lema } \\
\text { Figuras, (cornucopia, flores y frutos): "objetos } \\
\text { que se colocan en el campo del Escudo" } \\
\text { Fasces colombianas: conjunto de } 12 \text { flechas } \\
\text { unidas en un cilindro que son, sostenidas por la } \\
\text { presión de una segur o hacha atravesada. } \\
\text { Arcos y flechas: tres arcos y tres flechas cruzados } \\
\text { en diagonal hacia arriba y separados } \\
\text { Esmalte: "(...) colores con que se pinta tanto el } \\
\text { campo como las figuras del escudo" }\end{array}$ \\
\hline Función & $\begin{array}{l}\text { La función original del escudo de la Universidad } \\
\text { de Cartagena, fue la de identificar a la naciente } \\
\text { gran república ante el concierto de las naciones, } \\
\text { la Gran Colombia. Es decir que su función actual, } \\
\text { tiene un antecedente histórico de transferencia de } \\
\text { la identificación de la Gran Colombia a la identifi- } \\
\text { cación de la Universidad de Cartagena. }\end{array}$ \\
\hline
\end{tabular}

Fuente: Fotografías tomadas del proyecto de investigación: Universidad e identidad. Usos y apropiaciones de los signos visuales y representaciones sociales de la Universidad de Cartagena. Autores Indira Romero Peñaranda y Jorge Matson Carballo. 2013.
${ }^{1}$ Las palabras técnicas de la ciencia heráldica, fueron tomadas del estudio "Breve introducción a la heráldica" de Ignacio Gaviria Tomás. Académico correspondiente de la Academia Marínense de Heráldica y Genealogía. Se utilizó por su carácter pedagógico y de fácil manejo para los no iniciados en la heráldica. http:// www heraldicahispanica.com/IntroH. htm\# her 


\section{Usos de los signos visuales de la Universidad de Cartagena}

El análisis de usos de los signos visuales permitió la identificación de las siguientes variaciones en las aplicaciones de los signos visuales (ver figura 1). Acerca del escudo de la Universidad de Cartagena las inconsistencias en las aplicaciones del identificador institucional son constantes. Por otro lado, la mayoría de las veces el escudo se complementa con el logotipo Universidad de Cartagena y frecuentemente se encuentra además de las palabras Universidad de Cartagena, las palabras: fundada en 1827, o solo el año 1827.
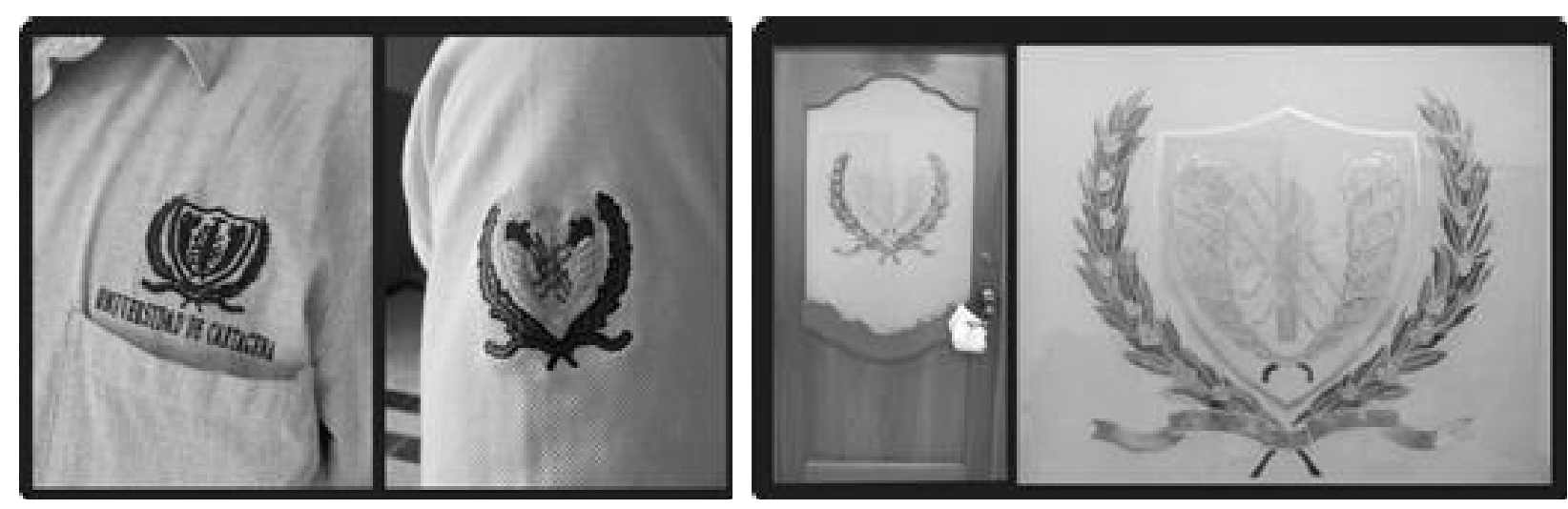

Fuente: Fotografías tomadas del proyecto de investigación: Universidad e identidad. Usos y apropiaciones de los signos visuales y representaciones sociales de la Universidad de Cartagena. Autores Indira Romero Peñaranda y Jorge Matson Carballo. 2013
Figura 1. Variaciones en el uso de los signos visuales.

Acerca de la imagen de la Torre: es recurrente en las aplicaciones de carácter comunicacional que se realizan en la Universidad de Cartagena. Es presentada es dos versiones, como imagen ilustrada como un dibujo vectorizado digitalmente y como imagen fotográfica. En ambas, el plano en contrapicado le imprime grandeza y poder a esta imagen, con lo cual se reafirma el carácter de solemnidad de la Universidad de Cartagena.
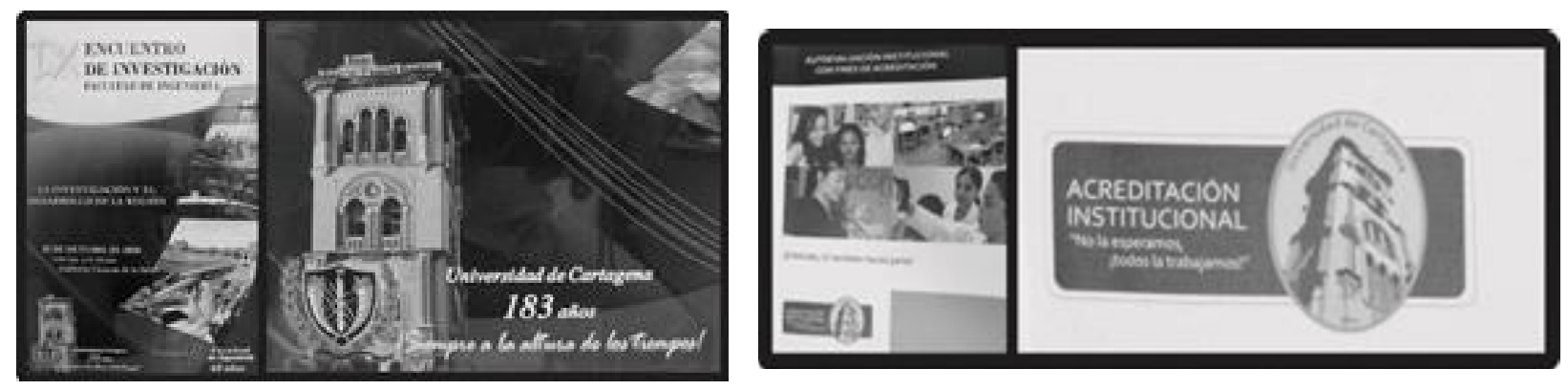

Figura 2. Imagen de la torre

En las aplicaciones cotidianas de los identificadores de la Universidad de Cartagena, se presentan otros. En un primer grupo, es posible apreciar todos los emblemas e identificadores de las distintas unidades académicas, 
especialmente facultades y programas académicos. Por lo evidenciado en las imágenes fotográficas del registro de esta observación, es fácil notar que cada uno es muy distinto del otro y no existen lineamientos estilísticos ni técnicos al respecto. El otro grupo de identificadores está dado por casos curiosos, que en el intento por conformar identificadores institucionales, parecieran ser iniciativas emergentes y sin la intención que perduren y se normalicen, como el identificador que usa la Torre en la portada de la publicación de autoevaluación (ver figura 2).

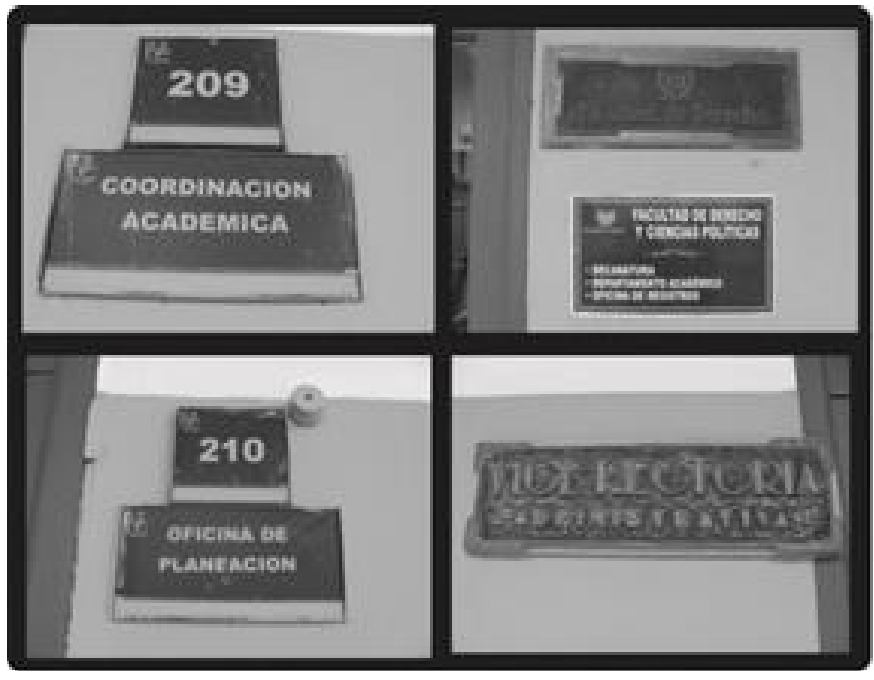

Figura 3. Otros identificadores

Fuente: Fotografías tomadas del proyecto de investigación: Universidad e identidad. Usos y apropiaciones de los signos visuales y representaciones sociales de la Universidad de Cartagena. Autores Indira Romero Peñaranda y Jorge Matson Carballo. 2013.

Tabla 5. Apropiación de los signos visuales y representaciones sociales

\begin{tabular}{|c|c|}
\hline $\begin{array}{l}\text { Significados conferidos } \\
\text { a la U de C }\end{array}$ & $\begin{array}{l}\text { Alma Máter: la gran madre, referente } \\
\text { arquitectónico, referente social: oportunidad de } \\
\text { educación, inclusión social. }\end{array}$ \\
\hline Sentimientos que evoca & $\begin{array}{l}\text { Agradecimiento, lealtad, compromiso, respeto, } \\
\text { familia, gloria, tradición y pertenencia. }\end{array}$ \\
\hline $\begin{array}{l}\text { Características que } \\
\text { identifican a la U de C }\end{array}$ & $\begin{array}{l}\text { Calidad académica, formación integral, } \\
\text { formación en investigación, libertad y crítica, } \\
\text { tradición e historia. }\end{array}$ \\
\hline $\begin{array}{l}\text { Identificación de signos visuales } \\
\text { que expresan identidad. }\end{array}$ & $\begin{array}{l}\text { Escudo de la Universidad de Cartagena, Torre del } \\
\text { Claustro de San Agustín, uniformes, monograma } \\
\text { U de C, colores amarilloy negro, color de la fachada, } \\
\text { la bandera de la Universidad de Cartagena. }\end{array}$ \\
\hline $\begin{array}{l}\text { Identificación de signos no } \\
\text { visuales que expresan identidad. }\end{array}$ & $\begin{array}{l}\text { Eslogan: A la altura de los tiempos e Himno } \\
\text { El Tropelín. }\end{array}$ \\
\hline $\begin{array}{l}\text { Sentimientos que evocan, } \\
\text { significados que se le confieren. }\end{array}$ & $\begin{array}{l}\text { Escudo de la Universidad de Cartagena: respeto, } \\
\text { abarcador, orgullo. Torre del Claustro de San } \\
\text { Agustín: imponencia, historia, pasado, faro. } \\
\text { Uniformes: calidad académica, reputación e } \\
\text { status. Colores amarillo y negro: Universidad } \\
\text { de Cartagena. Color de la fachada: Universidad } \\
\text { de Cartagena. La bandera de la Universidad de } \\
\text { Cartagena: ausencia. }\end{array}$ \\
\hline
\end{tabular}

Palobra No. 14. Agosto de 2014 
Fuente: Fotografías tomadas del proyecto de investigación: Universidad e identidad. Usos y apropiaciones de los signos visuales y representaciones sociales de la Universidad de Cartagena. Autores Indira Romero Peñaranda y Jorge Matson Carballo. 2013.

\begin{tabular}{|c|c|}
\hline $\begin{array}{l}\text { Identificación de la comunidad } \\
\text { universitaria con la imagen } \\
\text { que proyecta }\end{array}$ & $\begin{array}{l}\text { Reconocimiento externo, en el sector laboral. } \\
\text { Confianza de la comunidad. Superioridad. }\end{array}$ \\
\hline Actividades administrativas & $\begin{array}{l}\text { Los procesos de aseguramiento de calidad de los } \\
\text { programas y demás actividades propias de los } \\
\text { cargos administrativos. }\end{array}$ \\
\hline Actividades académicas & $\begin{array}{l}\text { Formación integral, la formación en investigación } \\
\text { y trabajo en equipo. }\end{array}$ \\
\hline Actividades culturales & $\begin{array}{l}\text { Proceso de elecciones, cumpleaños de la Universidad, } \\
\text { marcha de estudiantes y actividades deportivas. }\end{array}$ \\
\hline $\begin{array}{l}\text { Usos del escudo de la } \\
\text { Universidad de Cartagena }\end{array}$ & Papelería, presentaciones y afiches. \\
\hline Usos uniformes & $\begin{array}{l}\text { Uniformes de carrera, batas en el área de la salud, } \\
\text { uniformes de servicios generales, uniformes de } \\
\text { personal administrativo. }\end{array}$ \\
\hline $\begin{array}{l}\text { Significados conferidos al escudo } \\
\text { de la Universidad de Cartagena }\end{array}$ & $\begin{array}{l}\text { Hermosura, dignidad, tradición, historia, } \\
\text { institucional, uso libre, universidad, } \\
\text { indispensable, identidad, empoderamiento. }\end{array}$ \\
\hline $\begin{array}{l}\text { Significados conferidos } \\
\text { a los uniformes }\end{array}$ & $\begin{array}{l}\text { Posicionamiento de la imagen de la universidad, } \\
\text { profesionalismo, igualdad entre estamentos, } \\
\text { necesidad funcional, distinción. }\end{array}$ \\
\hline $\begin{array}{l}\text { Ícono: } \\
\text { Valor icónico, de semejanza } \\
\text { de los signos visuales }\end{array}$ & $\begin{array}{l}\text { La Torre: como lugar tradicional de la } \\
\text { Universidad de Cartagena. Como representación } \\
\text { del Claustro de San Agustín. Como monumento } \\
\text { arquitectónico. }\end{array}$ \\
\hline $\begin{array}{l}\text { Índice: } \\
\text { Valor indicativo, de señal, } \\
\text { de los signos visuales }\end{array}$ & $\begin{array}{l}\text { La Torre del Claustro de San Agustín como } \\
\text { referente de ubicación geográfica en el Centro } \\
\text { Histórico de Cartagena. Los colores y la forma de } \\
\text { su fachada son un referente de la ubicación de la } \\
\text { Universidad de Cartagena. }\end{array}$ \\
\hline $\begin{array}{l}\text { Símbolo: } \\
\text { Valor simbólico, cultural y } \\
\text { emocional de los signos visuales }\end{array}$ & $\begin{array}{l}\text { La Torre: poder, imponencia, sublime. } \\
\text { Tradición, historia y antigüedad. }\end{array}$ \\
\hline
\end{tabular}

\section{Conclusiones}

a.Se identificó, que si bien la Universidad de Cartagena se encuentra desarrollando un proceso de internacionalización, no consideró el elemento de la identidad como un insumo fundamental para poder enfrentar este reto. Sin embargo, es un hecho que una muy buena parte de su comunidad académica está consciente de la importancia de esta para alcanzar los objetivos propuestos en el proceso de autoevaluación para la acreditación 


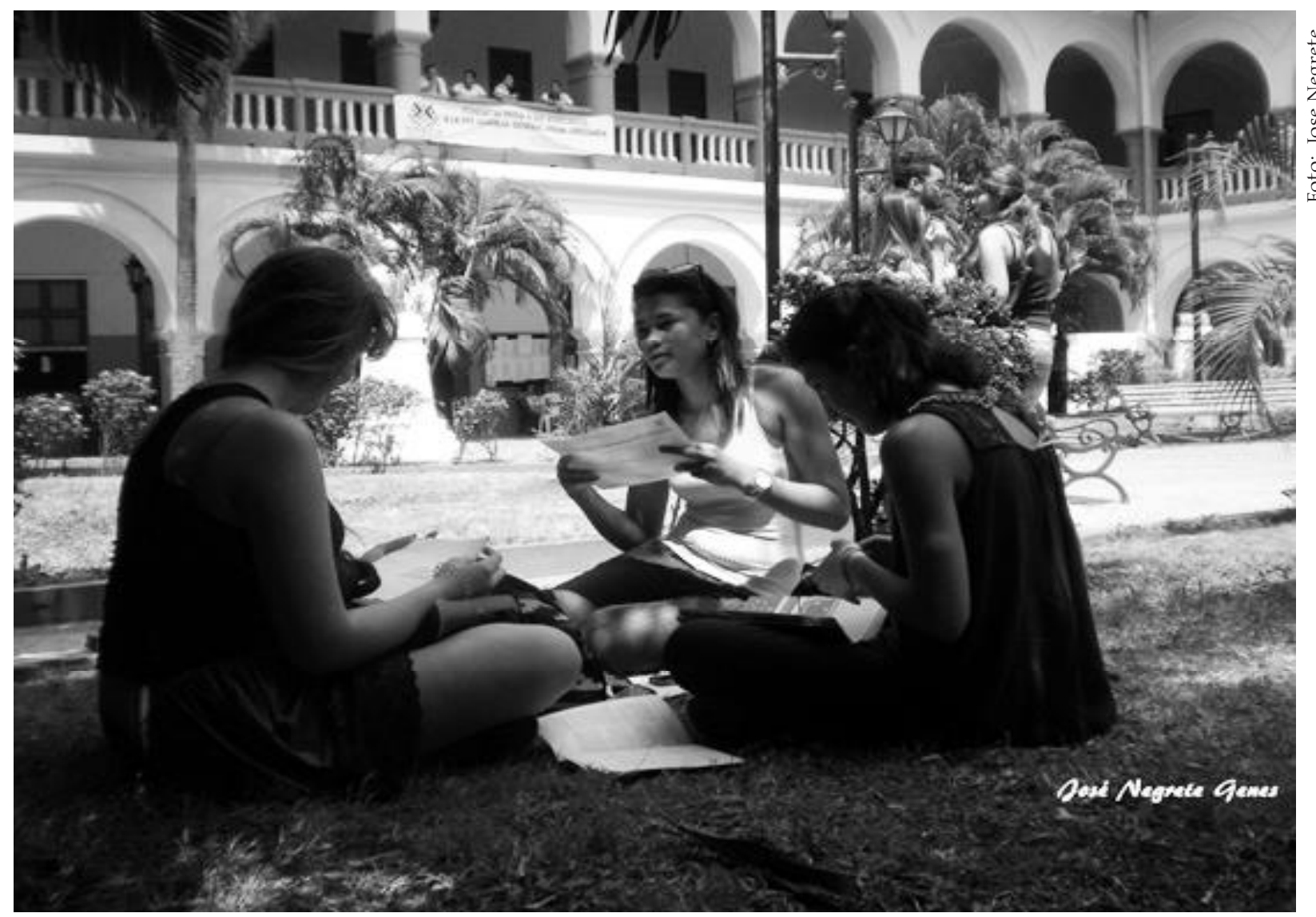

institucional. Efectivamente, al considerar, el CNA, como un elemento sustancial para la acreditación el reconocimiento de la identidad propia de las IES, la tradición histórica de la Universidad basada en la relación de origen con los ideales libertarios, de ser una fortaleza puede convertirse en una debilidad, si esa experiencia de reconocimiento no se considera en conjunto con otros aspectos sustanciales de la identidad como el uso de los signos visuales y la reconfiguración constante de las formas de apropiación y las representaciones sociales que se desprenden de esa dinámica.

b.En la Universidad de Cartagena no existe una práctica normalizada sobre el uso y aplicaciones de los signos visuales que la identifican. Aunque han empezado a esbozarse una política institucional que regule todos los aspectos fundamentales de su comunicación, con la elaboración de un primer Plan de Comunicación, en la actual administración, aún no se hace visible, en la cotidianidad, un acuerdo mínimo de las aplicaciones prácticas de estos signos visuales.

c.Como signos visuales representativos para la comunidad universitaria, emerge

Palobra No. 14. Agosto de 2014 
"la torre, sin embargo cuando aparece el escudo, este es valorado como más representativo porque puede ser visto y presentido por su carácter omnisciente en cada instancia en donde las prácticas sociales y culturales de la Universidad se hacen manifiestas, indistintamente si se está en el Claustro de San Agustín o se representa a la Universidad de Cartagena en actividades externas o de internacionalización, de carácter académico, investigativo o simplemente en el transitar por la ciudad de Cartagena en actividades cotidianas.

d.La mención del nombre Universidad de Cartagena, no solo remite al reconocimiento de la torre y el escudo como símbolo, se genera también una imagen mental sobre el claustro, sobre todo entre los representantes de estamentos que han vivido sus experiencias cotidianas en ese sitio la mayor parte de su vida activa.

e.El uso indiscriminado de los signos visuales, en especial del escudo, ha favorecido una inadecuada apropiación, en el sentido de interpretarse esto como la libertad que tiene el Udeceista para utilizarlo como, cuando, donde y en las condiciones que le parezca, pues así expresa su pertenencia asociado a su personalidad, permeada esta por lo disciplinar. Ante esa inapropiada mirada y valoración de uso del identificador primario, la Universidad de Cartagena se enfrenta con un delicado problema de interacción con su entorno, puesto que la regulación de la imagen de los organizaciones (incluidas la IES) por medio de manuales de identidad o de imagen corporativa es una constante en el mundo para facilitar la comunicación en la era del signo.

f.El valor de ícono, de índice y de símbolo de los signos visuales de la Universidad de Cartagena están presentes de manera integrada, por lo cual no son excluyentes, tal como lo demuestra el uso remitidos a los mismos. En consecuencia, la constitución del signo es inseparable del análisis, es su resultado, ya que, sin él, no aparecería. [...] " Es también su instrumento, ya que, una vez definido y aislado, puede ser remitido a nuevas impresiones; [...] El signo aparece porque el espíritu analiza. El análisis prosigue porque el espíritu dispone de signos." (Foucault, 2007, p. 67.), En este sentido es importante ratificar que cuando se habla de signo visual, no se hace referencia a la representación visual que aparece ante los ojos, se habla de signo cuando a través de la experiencia previa se establece una relación entre el objeto y la persona se conforma un sentido producto de esa relación.

g.Los significados que se le confieren a la Universidad de Cartagena, son los mismos que se le dan a sus signos sean estos visuales o no, (relación 
de mutualidad). El escudo es signo, solo cuando aparece como elemento de una representación social, cuando se establece una relación entre sus dimensiones espirituales y prácticas, es decir los usos y aplicaciones por sí solas no hacen al escudo un signo, solo cuando hay apropiación, cuando se le confiere un significado, es posible hablar de un signo. Es decir que los signos visuales y las representaciones sociales configuran identidad en una relación interdependiente, en la cual existe una circulación y actualización de significados y sentidos.

\section{Recomendaciones}

a. Fortalecer las acciones para consolidar la identidad institucional entendida como una configuración que se alimenta de los actos cotidianos y tanto de la dimensión racional como la emocional de las personas que hacen parte de la comunidad universitaria, cuestión que es posible abordar desde una evaluación permanente de la cultura institucional por medio de un asertivo programa de

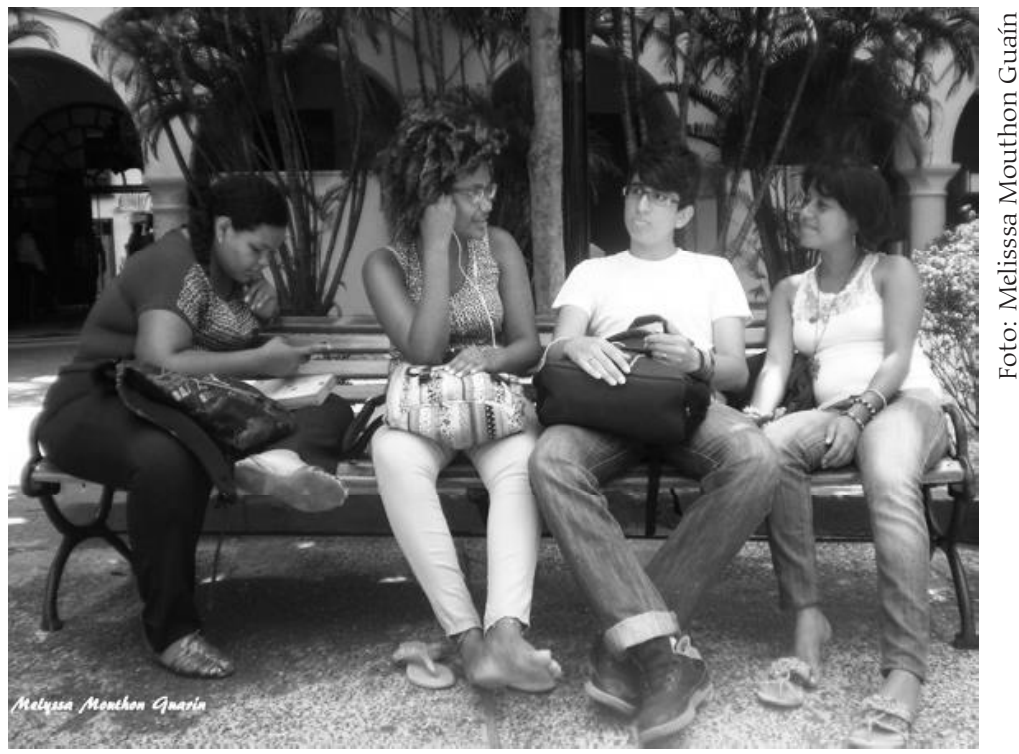
comunicación interna.

b.Diseñar un plan de comunicaciones que contemple lo estratégico de la comunicación desde los diferentes frentes de la internacionalización, pero ante todo que se preocupe de establecer el estado de la situación interna de usos y apropiaciones de los signos visuales, representaciones sociales y demás aspectos de la identidad, para que se traduzca en instrumentalización estratégica de la imagen a partir de un manual de identidad institucional.

c.Desarrollar actividades culturales, organizacionales y de bienestar, que generen pertenencia institucional a través de la participación como complemento del proyecto "Cátedra de la Historia de la Universidad de Cartagena. Mirar su historia para construir su futuro". Es decir implementar y mantener experiencias de socialización, como complemento a las curriculares, que por interacción directa con la comunidad universitaria permita reflexionar sobre las políticas internas relacionadas con los aspectos axiológicos, históricos y culturales de la Universidad. 


\section{BIBLIOGRAFÍA}

1. Augé, M. (1996). El sentido de los otros. Actualidad de la antropología. España: Paidos.

2. Chevalier, J., \& Gheerbrant, A. (1999). Diccionario de símbolos. España: Herder.

3. Consejo Nacional de Acreditación (CNA). (s.f.). Procesos de Acreditación. Acreditación Institucional. Obtenido de http://www.cna.gov.co/1741/article-186371.html

4. De la Garza , E. (Enero - Marzo de 2001). La epistemología crítica y el concepto de configuración. Revista Mexicana de Sociología., LXIII(1), 109-127.

5. Dilthey, W. (1978). El sueño de Dilthey. Documentos autobiográficos, en Introducción a las ciencias del espíritu. México: Fondo de la Cultura Económica.

6. Foucault, M. (2007). Las palabras y las cosas, una arqueología de las ciencias humanas (13 ed.). (E. C. Frost, Trad.) Siglo XXI editores.

7. Hall, S. (2003). Introducción: ¿Quién necesita identidad? En Cuestiones de identidad cultural. Buenos Aires: Amorrotu Editores.

8. Gonzalez Ramírez, T. (2005). El proceso de convergencia europea y la identidad de las universidades en la Europa del conocimiento. Revista Fuentes 6. Universidad de Sevilla.

9. Jodelet, D. (1976). La representación social: fenómenos conceptos y teoría. En S. Moscovici, Pensamiento y vida Social. Sociopsicologia.files.wordpress.com/2010/05/rsociales-djodelet.pdf: Paidós.

10. Matson Carballo, J. (2004). El escudo de la Universidad de Cartagena: Símbolo de identidad. En D. Piñeres De La Ossa, La Cátedra de la Universidad de Cartagena. Mirar su historia para construir su futuro. Cartagena: Editorial Universitaria.

11. Ministerio de Educación Nacional. (2007). Tendencias de la educación superior en el contexto internacional., Revolución educativa: Educación Superior, boletín informativo \#9.

12. Moscovici, S. (2002). La representación social, un concepto perdido. En El psicoanálisis, su imagen y su publico (Segunda ed., págs. 27-44). Buenos Aires: Huemul. IEP - Instituto de Estudios Peruanos Taller Interactivo.

13. Organización de las Naciones Unidas para la Educación la Ciencia (UNESCO). (2008). Declaración de la conferencia regional de la educación superior en América Latina y el Caribe CRES. IESALC- Instituto Internacional de la UNESCO para la Educación Superior en América Latina y el Caribe.

14. Peirce, C. S. (2005). El ícono, el índice y el símbolo. (c. 1893-1903). Obtenido de Universidad de Navarra, Grupo de estudios peircianos: http://www.unav.es/gep/IconoIndiceSimbolo.html 